\title{
A Unity3D-based mobile fashion coordination system
}

\section{Daejin Kim, Wooyeol Ryu, Seungho Lee, Byungsu Lim and Sangjun Lee*}

School of Computing,

Soongsil University,

Seoul, 06978, Korea

Email: terry915@naver.com

Email: rwy89@naver.com

Email: sbo78@naver.com

Email: bslim511@ssu.ac.kr

Email: sangjun@ssu.ac.kr

${ }^{*}$ Corresponding author

\begin{abstract}
The size of the fashion market is continuously growing and the number of worldwide mobile application users is increasing as well. Following this trend, a considerable number of mobile fashion applications have been developed and released; however, these applications simply provide various mix-and-match options and do not have other functions that consider user convenience. Unity3D is a game development ecosystem, in which three-dimensional (3D) and two-dimensional (2D) game contents can be created. Because its development is supported for various platforms such as Android, iOS and Blackberry, a 3D environment can be easily implemented in mobile devices. Using this environment, we can provide 3D virtual avatars to users along with an environment for intuitive access to the information that cannot be expressed in 2D. In this paper, an Android-based application is proposed to provide mix-and-match options customised according to the user's current wardrobe and the weather, and display these clothes on a virtual avatar via Unity3D.
\end{abstract}

Keywords: Unity3D; mobile; fashion; coordination.

Reference to this paper should be made as follows: Kim, D., Ryu, W., Lee, S., Lim, B. and Lee, S. (2016) 'A Unity3D-based mobile fashion coordination system', Int. J. Advanced Media and Communication, Vol. 6, No. 1, pp.86-92.

Biographical notes: Daejin Kim received his BS in School of Computing from Soongsil University, Seoul, South Korea in 2015. His current research interests include mobile systems and multimedia systems.

Wooyeol Ryu received his BS in School of Computing from Soongsil University, Seoul, South Korea in 2015. His current research interests include mobile systems and multimedia systems.

Seungho Lee received his BS in School of Computing from Soongsil University, Seoul, South Korea in 2015. His current research interests include web services and information security. 
Byungsu Lim received his MS and BS in School of Computing from Soongsil University, Seoul, South Korea in 2014 and 2016, respectively. His current research interests include database systems and cloud computing.

Sangjun Lee received his $\mathrm{PhD}$ in the School of Electrical Engineering and Computer Science, Seoul National University, Seoul, South Korea in 2004. $\mathrm{He}$ is currently an associate professor of the School of Computing, Soongsil University, Seoul, South, Korea. His current research interests include database systems, mobile systems and cloud computing.

This paper is a revised and expanded version of a paper entitled 'A Unity3Dbased intelligent mobile fashion coordination system' presented at 2015 International Conference on Platform Technology and Service, Jeju, Korea, 26-28 January, 2015.

\section{Introduction}

Fashion in today's society has emerged as an item that expresses a person's personality. As shown in Table 1, the global apparel market in MarketLine (2013) has been growing continuously from USD 1249.3 billion in 2012 and is expected to reach USD 1562.6 billion in 2017. Furthermore, as smartphones are widely used globally, the field of mobile applications has become essential. According to Portio Research (2015), as shown in Table 2, the number of worldwide mobile application users was forecasted to increase from 740 million in 2011 to 2.4 billion in 2014 .

Table 1 Global apparel market size in MarketLine (2013)

\begin{tabular}{lcc}
\hline Year & Market value of apparel industry (USD Billion) & Growth rate $(\%)$ \\
\hline 2012 & 1249.3 & 3.1 \\
2013 & 1300.9 & 3.1 \\
2014 & 1357.0 & 4.1 \\
2015 & 1418.9 & 4.6 \\
2016 & 1487.4 & 4.8 \\
2017 & 1562.6 & 5.1 \\
\hline
\end{tabular}

Table 2 Global mobile application users in Portio Research (2015)

\begin{tabular}{lccccccc}
\hline & 2011 & 2012 & 2013 & 2014 & 2015 & 2016 & 2017 \\
\hline No. of users (million) & 704 & 1186 & 1752 & 2400 & 3068 & 3761 & 4367 \\
\hline
\end{tabular}

Summarising Tables 1 and 2, we find that the global apparel market and the number of worldwide mobile application users are increasing. Accordingly, an increase in the demand for mobile fashion applications is expected in Kim (2011). To supplement the disadvantages of conventional mobile fashion applications and increase user convenience, in this paper, we propose a mobile fashion application that provides customised mix-and-match options based on the weather, gender and the user's existing 
wardrobe, and provides a function that can display the recommended options on a three-dimensional (3D) avatar using Unity3D.

This paper is organised as follows. In Section 2, background is described. In Section 3, our proposed system is described. In Section 4, a conclusion for this study is presented.

\section{Background}

In this section, we will review the fashion applications available in the market and describe the technologies required for mobile application-based fashion coordination recommendation and $3 \mathrm{D}$ avatar.

\subsection{Analysis of conventional fashion applications}

Typical fashion applications are Pose (2015) and Netrobe (2015). The Pose application classifies fashion styles into many items and has a purchasing function that links with an online shopping mall, but it just shows various styles and does not have a recommendation function. The Netrobe application is a fashion SNS application in which a user can take photographs of clothes and upload them. Like Pose, Netrobe does not have a function that provides recommendations related to the clothes owned by the user.

\subsection{Unity $3 D$}

Unity3D (2015) is a game development ecosystem in which 3D and two-dimensional (2D) game contents can be created. Since Unity3D supports multiple platforms, games can be developed on various platforms such as iOS, Android and Blackberry. Using Asset Store, the user can use assets created by other users by paying a certain fee when Unity3D is used as a game engine.

\section{Proposed system}

In this paper, we design and implement a Unity3D-based Mobile Fashion Coordination System to provide mix-and-match options customised according to the user's current wardrobe and the weather, and display these clothes on a virtual avatar via Unity3D. Proposed system was developed by using Android 4.0.3 (Ice Cream Sandwich) SDK on Windows 7 SP1. MySQL was used for the database and the server was set up by using Apache Tomcat Server v7.0. To be able to display clothes on a virtual mannequin, Unity3D was linked with Android.

\subsection{Overall system architecture}

In this paper, overall system architecture is shown in Figure 1. The proposed system is largely made up of Server and Client. The server side is responsible for processing the client requests and storing the information which is required to recommend clothes for users. As shown in Figure 1, the server consists of a web server, database server and public data collection server. The web server provides functions such as the coordinate 
search using location information and coordinate recommendation search using the recommendation algorithms in communication with the client application. The database server stores the member information and the mix-and-match options. Our system is also designed to be accessed through a servlet for database for increased security. The public data collection server periodically collects weather information from public data servers operated by the government. The proposed system provides a coordinate for the weather to the user based on the collected weather information. The client refers to a mobile application based on android and provides various functions to connect to the server. The client consists of a setup part for managing location information and member information, and an image part for checking a recommended mix-and-match option and displaying it on the virtual avatar.

Figure 1 Overall system architecture

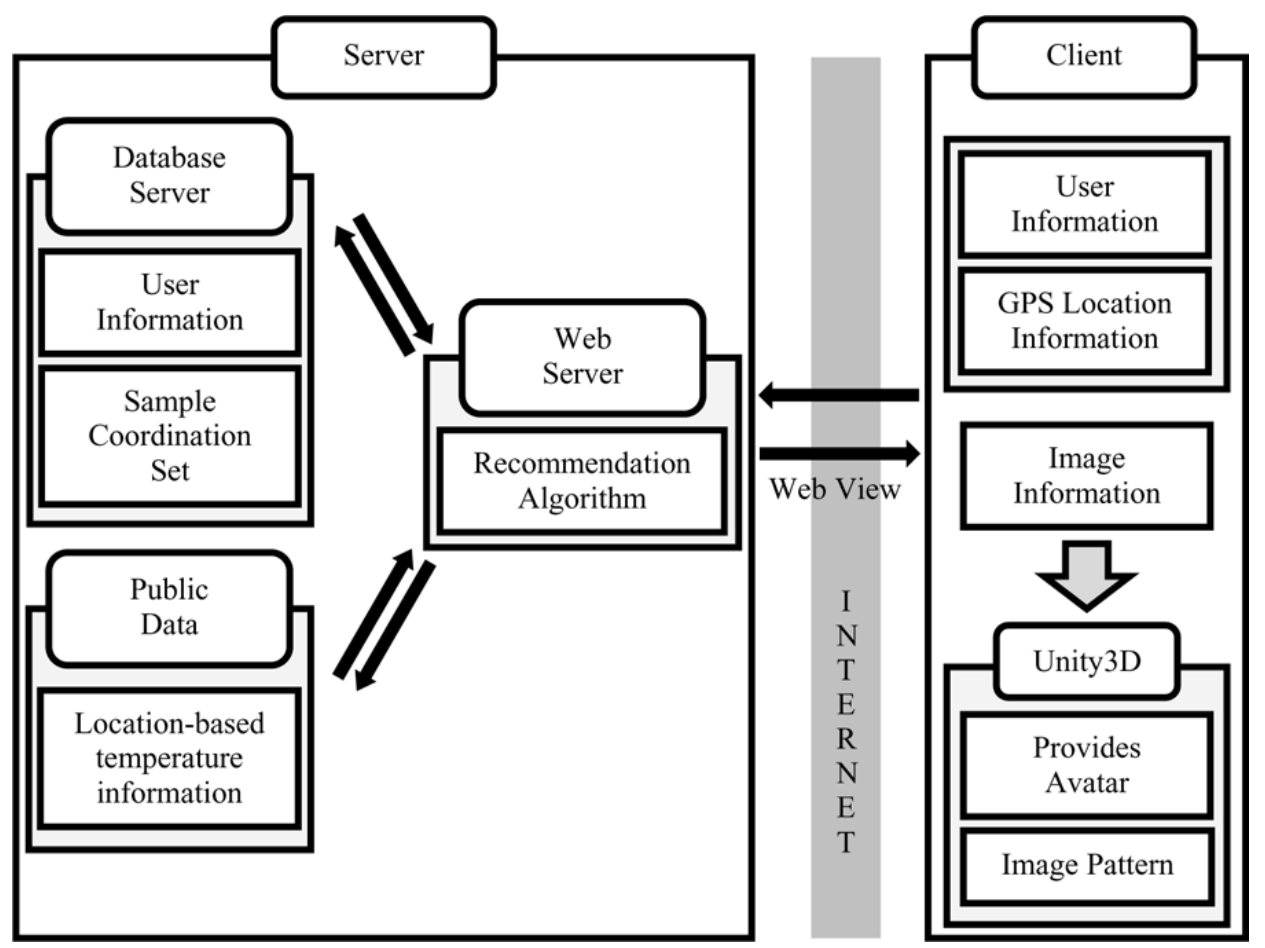

\subsection{Production process of $3 D$ s max}

The proposed system is implemented for the modelling of gender-specific body shapes using 3Ds Max (2015). An avatar has to be constructed by modelling the types of recommend clothes in advance. The clothes modelled as 3D object types export the FBX format files. And these files are used Android application through the Unity3D.

Upon inputting the option values for clothes, the user obtains a 3D avatar wearing the applicable clothes. Since the avatar can be rotated freely, the user can see the recommended outfit from various angles. Figures 2 and 3 show the final modelling of a female T-shirt and one-piece dress in 3Ds Max, respectively. 
Figure 2 Female T-shirt modelling using 3Ds Max (see online version for colours)

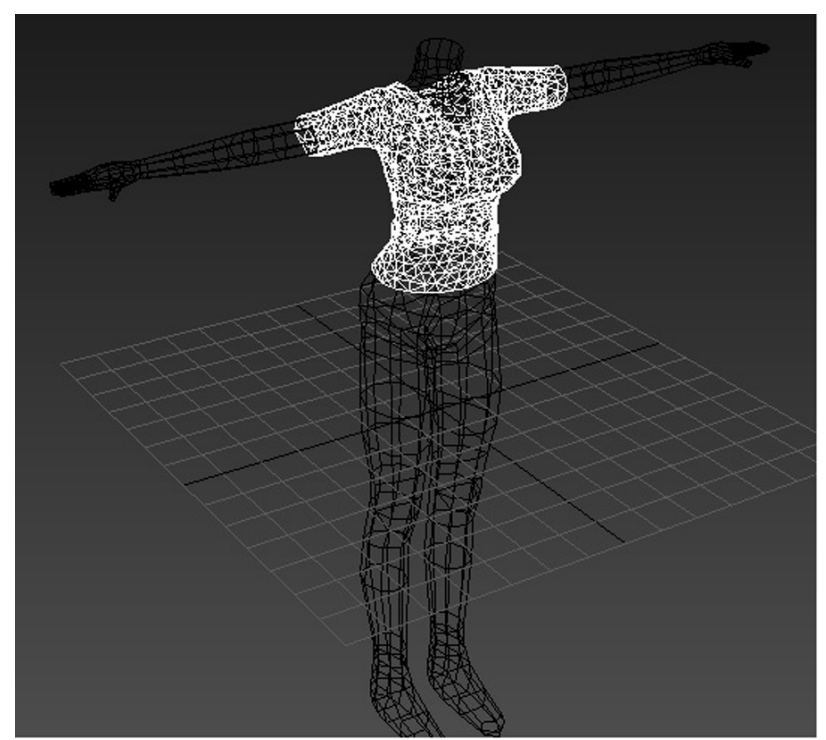

Figure 3 Female one-piece modelling using 3Ds Max (see online version for colours)

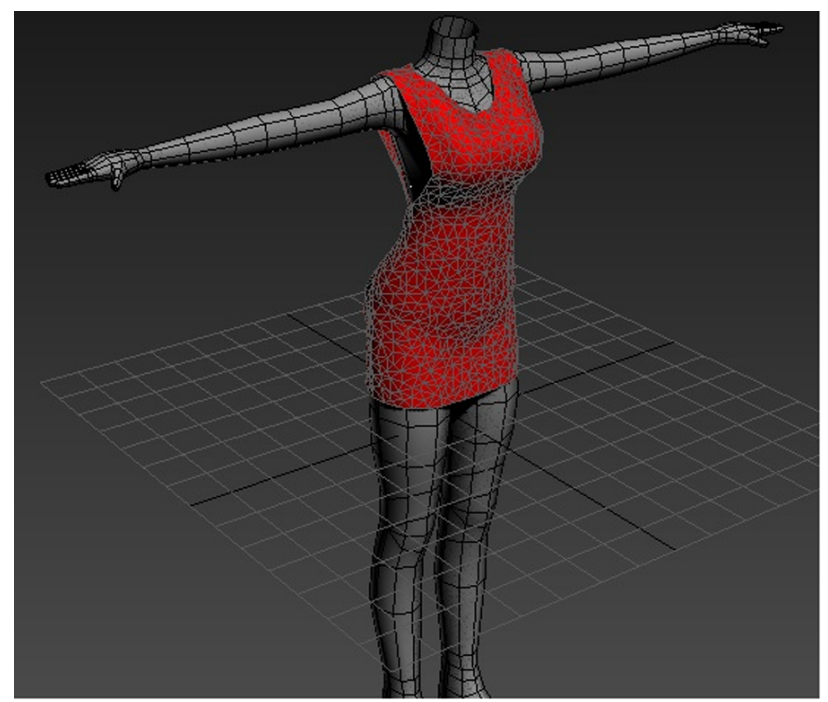

\subsection{Providing recommended fashion options}

Users receive fashion coordination on the main screen of the application according to weather and gender. Figure 4 shows the results of recommended fashion coordination on the main screen. Users can choose the colours and types of clothes based on the option values. The application compares the coordination samples stored in the database according to the options chosen by users and shows total 10 samples that appear to be the most proper. 
Figure 4 Example of recommended fashion coordination (see online version for colours)

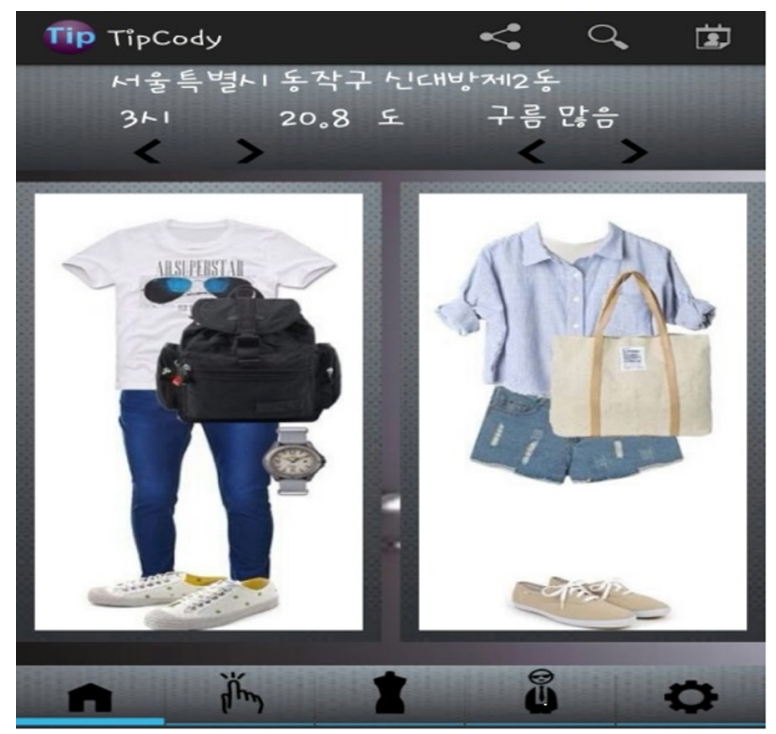

Weather data are updated every $3 \mathrm{~h}$ with public data by the server, and the user receives a fashion option suitable for the weather and the gender on the main screen of the application. Furthermore, the user selects the desired colours and type of clothes as option values. According to the selected options, the mix-and-match options stored in the DB server are compared, and 10 most suitable images are recommended; then, the user can view the recommended fashion options on the 3D avatar. Figure 5 shows the recommended clothes on an avatar.

Figure 5 Unity3D virtual Avatar (see online version for colours)

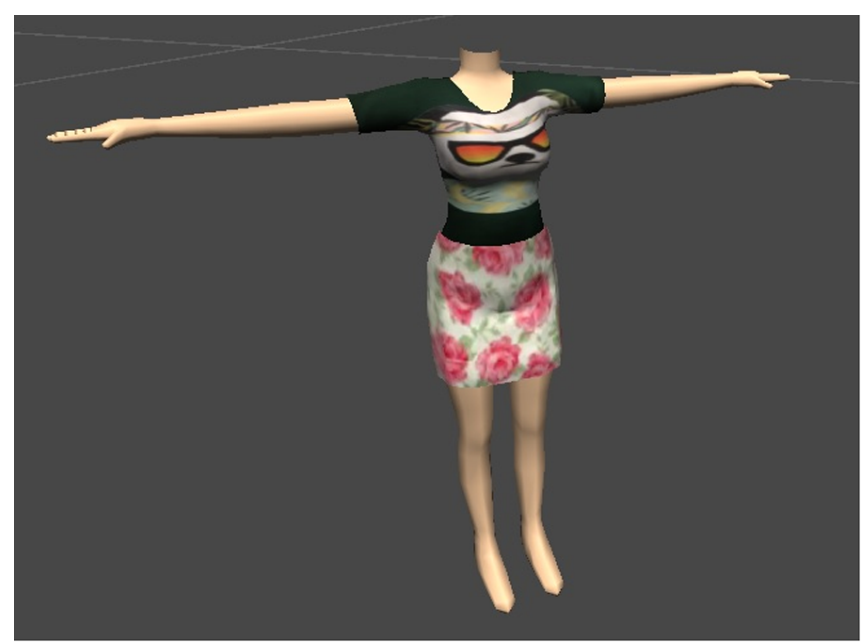




\section{Conclusion}

The fashion applications available in the market do not provide customised information to users because of their commercial tendencies. First, a recommendation service based on the user's wardrobe and the weather is lacking. Secondly, users can receive various fashion recommendations, but there are few applications with functions that can actually match user's clothes to a fashion or provide customised fashion information appropriate for a certain day. In this paper, an Android-based application was proposed to provide customised fashion options according to the user's wardrobe and the weather, and display the recommended clothes on a virtual avatar via Unity3D. The deficiencies of conventional fashion applications were overcome by creating three functions, namely, a function that provides fashion recommendations customised according to the weather, where information was obtained from public data, to the user; a function that provides clothes recommendation based on the clothes owned by the user; and a $3 \mathrm{D}$ virtual avatar function that provides more intuitive information than a $2 \mathrm{D}$ photograph. The released Android applications are single-platform applications. However, in this paper, a multi-platform application was proposed, which combines the Android platform and the Unity3D game engine's platform. Future fashion applications developed using sophisticated image processing and rendering systems will play a critical role in the mobile fashion market. In future, users will receive various services through multi-platforms rather than services obtained through a single platform.

\section{Acknowledgements}

This research is supported by the MSIP (Ministry of Science, ICT and Future Planning), Korea, under the Seoul Accord Vitalisation Program (IITP-2015-R0613-15-1175) supervised by the IITP (Institute for Information and communications Technology Promotion).

\section{References}

3Ds Max (2015) http://www.autodesk.co.kr/products/3ds-max/overview (Accessed 21 March, 2015).

Kim, Y. (2011) 'A study on effects of mobile properties and involvement on satisfaction of smartphone application: focused on fashion application', The e-Business Studies, Vol. 12, No. 2, pp.49-77.

MarketLine (2013) Global Apparel Retail, http://www.marketline.com (Accessed 21 March, 2015).

Netrobe (2015) https://netrobe.com/ (Accessed 21 March, 2015).

Portio Research (2015) (Accessed 21 March, 2015).

Pose (2015) https://pose.com (Accessed 21 March, 2015).

Unity3D (2015) http://unity3d.com (Accessed 21 March, 2015). 